\title{
ESTIMATION OF PROTHROMBIN IN DICOUMARIN THERAPY
}

\author{
BY \\ ROSEMARY BIGGS AND R. G. MACFARLANE \\ From the Department of Pathology, Radcliffe Infirmary, Oxford
}

(RECEIVEd FOR PUbliCATION, SEPTEMBER 8, 1948)

The concept of prothrombin as the specific precursor of thrombin dates from the so-called classical theory of blood coagulation put forward by Schmidt (1895) and Morawitz (1905). This theory, which considers that four factors, thromboplastin, prothrombin, calcium, and fibrinogen, are concerned in the formation of fibrin, has been the basis of most modern work. The theory was of more academic than practical importance until the discovery of vitamin $\mathbf{K}$ and the use of dicoumarin in treatment of thrombosis, both of which required the quantitative estimation of prothrombin. The available methods are all based on the assumption that the classical theory is fundamentally sound.

Of the methods of prothrombin estimation that have been developed, none can measure prothrombin directly and therefore infer its concentration from observations on thrombin. The one-stage technique of Quick (1942) has been the most commonly used because it is simple, easily performed, requires little in the way of special reagents, and gives results which are in general agreement with clinical experience. The method consists of adding an optimum amount of brain thromboplastin and calcium to oxalated plasma, under which conditions the clotting time of the mixture is assumed to be proportional to its prothrombin concentration. The basis of this supposed relationship is the literal acceptance of the classical theory. If three of the four variables are controlled, then variations in the fourth must determine any alteration in the rate of fibrin production, which is in turn indicated by a change in the clotting time of the mixture. In practice thromboplastin and calcium are controlled, if possible at or near their optimum concentrations, and fibrinogen variations found in the ordinary clinical material are usually not of sufficient magnitude to influence the result. The remaining variable is therefore prothrombin.
The method is essentially dynamic in principle, depending entirely on the rate of thrombin generation and not on the amount of thrombin that may finally be produced. It depends also on the assumption that there are no uncontrolled factors other than prothrombin that might modify this rate of thrombin generation. It is now known, of course, that this is not the case. Not only may the reaction time of fibrinogen vary, but accelerators and depressors of thrombin generation are present in normal plasma, and variation in these accelerators and depressors in pathological conditions may alter the results of this test. Quick (1942) himself has been the first to recognize this. The practical implications of the fallacious nature of this method are difficult to assess at present, but no alternative to it is available for routine use. Though theoretically the two-stage method appears to be on much firmer ground, since it seeks to estimate the total amount of thrombin generated, it is too complex for ordinary routine use. There are, moreover, many indications that its results may be equally fallacious.

In dicoumarin therapy one component of prothrombin (prothrombin B of Quick, 1947) is deliberately lowered, and the responsibility for a reduction to haemorrhagic levels rests largely with the pathologist. A reliable method of prothrombin determination is therefore essential. There has recently been much discussion as to which of the modifications of the one-stage technique give the best results (Marsh, 1947, 1948 ; James, 1948 ; Cleland, 1947 ; Pivawar, 1947 ; Lempert, 1948 ; Canti and Robertson, 1948).

\section{Quick's Technique using Brain Thromboplastin in Dicoumarin Therapy}

Quick's technique using brain thromboplastin has been used to follow the effects of dicoumarin 
administration in numerous animal experiments and human cases (Bingham and others, 1941 ; Butsch and Stewart, 1942; Meyer and others, 1942; Shapiro and others, 1943; Barker and others, 1945 ; Bingham and others, 1943 ; Gefter and others, 1944; Barker and others, 1943 ; Jaques and Dunlop, 1945a ; Quick, 1945 ; Cotlove and Vorzimer, 1946; Allen, 1947 ; Glueck and others, 1948 ; etc.). In dogs single doses of $5 \mathrm{mg}$. per kilo can be relied upon to cause a fall in prothrombin to 10 per cent in 24 to 48 hours. In Allen's (1947) series of 1,686 cases a dose of $300 \mathrm{mg}$. was given on the first day and $200 \mathrm{mg}$. on the second (equivalent to 5 to $8 \mathrm{mg}$. per kilo); there was a fall in prothrombin within 24 to 72 hours. Other authors recommend a similar dosage and their cases followed the same general course. In a series of a thousand cases Barker and others (1945) claimed that haemorrhage was uncommon when the level of prothrombin was above 10 per cent (indicated by a lengthening of coagulation time from 19 to 60 seconds). A similar "danger level" has also bzen recorded in vitamin $\mathrm{K}$ deficiency. There is, therefore, evidence that prothrombin estimation by this method is in general agreement with the clinical manifestations of hypoprothrombinaemia.

\section{One-stage Technique using Russell's Viper Venom as Thromboplastin}

Modifications of the technique in use in this country involve the replacement of brain thrombop'astin with Russell's viper venom (Fullerton, 1940 ; Page and Russell, 1941 ; Page and others, 1941-2a and b ; Page and de Beer, 1942-3 ; Page and de Beer, 1943; Shapiro, Sherwin, Redish, and Campbell, 1942) or Russell's viper venom and lecithin (Witts and Hobson, 1940 and 1942 ; Hobson and Witts, 1941). It has been recognized for many years that certain snake venoms are powerful coagulants, and Martin (1894) was one of the first to investigate their action. Some, such as the venom of Echis carinatus and Notechis scutatus, are thrombin-like in nature as they clot oxalated blood. Lamb (1903) observed that Russell's viper venom was almost devoid of this thrombin-like effect, and the immensely powerful thromboplastin-like action of the venom was largely overlooked until Macfarlane -and Barnett (1934) reinvestigated its possibilities, in their search for a local haemostatic effective in haemophilia. It was found that this venom was capable of accelerating the coagulation of haemophilic blood in dilutions as low as 1 in $10^{12}$ or more, but was incapable of clotting oxalated or citrated blood or fibrinogen solutions. It was thought, therefore, to act as a thromboplastin, a view in accord with $\overrightarrow{\vec{F}}$ Mellanby's (1909) conclusions as to reactions of other viper venoms.

Following its use as a local haemostatic, Russell's $\frac{\bar{F}}{\bar{c}}$ viper venom was made available as a commercial $\stackrel{\varnothing}{\varnothing}$ product under a number of trade names. It was obtainable as a dry, sterile, and very stable prepara- $-\infty$ tion of tested potency in sealed ampoules. Such $\vec{\circ}$ preparations have obvious advantages over the $\overrightarrow{\vec{H}}$ more usual brain thromboplastin employed in Quick's original technique, as this is often difficult to obtain, not very stable, and of widely varying potency. These advantages were recognized byFullerton (1940), who suggested the use of venom as substitute for brain thromboplastin. The useo of Russell's viper venom as a substitute for brain? is only justified, however, if it is shown to behave 7 in all significant respects like the natural thrombo-응 plastin. Several observations have suggested that this is not the case. Trevan and Macfarlane (1936) found that the action of the venom was greatly $\overrightarrow{0}$ potentiated by tissue extracts and by use of lecithin. $\frac{+}{0}$ Macfarlane (1938) observed that plasma deprived of platelets by high-speed centrifuging was onlyo slowly clotted by venom, while Macfarlane and others (1941) showed that the removal of lipoido from the plasma inhibited its coagulation by venom, but this could be restored by the addition of various lipoid substances includ- $\overrightarrow{\vec{O}}$ ing lecithin. It appeared, therefore, that some 3 lipoid co-factor was essential to the action of the? venom, and it was thought with Leathes and? Mellanby (1939) that natural thromboplastin might consist of an enzyme and lipoid co-factor:analogous to Russell's viper venom and lecithin. Theoretically, therefore, the venom correspondso only to a part of the complete thromboplastin. $\frac{1}{3}$ In 1940 Witts and Hobson suggested the use of venom and lecithin mixture as a thromboplastin? for the one-stage method as being more nearlyo related to brain and providing a more rapid conversion of prothrombin. Such a mixture shortens $N$ the prothrombin time to 6 or 7 seconds.

There are thus obvious differences in actis between venom and natural thromboplastin even the supposition that the venom corresponcis a part of such a thromboplastin is unproved. It is therefore surprising that its use as a substitute for brain with or without the addition of lecithinfo has becoms widespread, particularly in England, without an adequate series of determinations in parallel with brain. Fullerton (1940) compared庆 Russell's viper venom and brain in 43 cases, but $\stackrel{\circ}{\sigma}$ gives no figures in his paper. Page and Russelk 
(1941) made a similar study in 71 cases, but few had any gross prothrombin deficiency and the coagulation times as presented cannot be assessed in relative percentage of prothrombin. Witts and Hobson (1940) tested the venom and lecithin method in 40 cases, but only two of these had prolonged prothrombin times, and Page and others (1941-2b) noted that the method failed to record minor degrees of prothrombin deficiency, evident when the venom alone was used.

With the advent of dicoumarin therapy it became more obvious that venom and lecithin did not give results comparable to those obtained with brain. Witts (1942) estimated the prothrombin time in a dog following a large dos? of dicoumarin and found that both with venom alone and with venom and lecithin the apparent decrease in prothrombin was less than was the case if brain was used for the estimations, an observation supported by de Beer (1947) for venom alone. Wright and Prandoni (1942), using venom for prothrombin determinations, found it necessary to give much larger doses of dicoumarin to achieve a reduction in prothrombin than were advised by other workers using brain for prothrombin estimation. In one case as much as $8,000 \mathrm{mg}$. were given. Moreover, the apparent fall in prothrombin was delayed from eight to ten days and eight serious haemorrhages occurred in the first 20 cases treated. Shapiro, Sherwin, and Gordimer (1942), and Shapiro and Sherwin (1943) made a similar study, but in only two cases were the aciual prothrombin times recorded. In one of these, $700 \mathrm{mg}$. were given in the first two days and there was a fall in prothrombin to 30 per cent (as estimated from our dilution curves) on the fifth day. In the second there was a fall to 15 per cent following $100 \mathrm{mg}$. of dicoumarin for six days.

'The scanty evidence from the literature therefore suggests that modifications of Quick's technique using Russell's viper venom with or without lecithin may give a less sensitive estimation of prothrombin deficiency. This evidence is supported by the two cases under treatment with "irsumarin recorded by Lempert (1948). In the 1.. If these Quick's technique gave 10 per cent nuarombin, where by Fullerton's technique the .evel was 35 per cent. In the second case, Fulleron's technique showed 25 per cent prothrombin "nd Quick's technique 10 per cent. A series of zases recently treated in this hospital supports the findings of Lempert, and suggests that Witts and Hobson's technique may give a dangerous impression of security in following the course of dicoumarin therapy.

\section{Technique}

(a) Quick's MethoD

1. Collection of blood.-Venous blood is collected in a dry syringe and added to 2 per cent potassium. oxalate solution in the proportion of nine paris of blood to one part of oxalate. The mixture is inverted several times and the plasma removed after centrifuging at 1,500 r.p.m. for 15 minutes. The plasma should be kept in the refrigerator and used within 6 hours of collection because the coagulation time of pathological plasmas may alter rapidly on storage.

2. Preparation of thromboplastin.-Fresh human brain is collected from the post-mortem room, all superficial vessels and meninges are removed, and the substance is macerated with acetone in a mortar. The acetone is. rep.aced several times and the granular powder dried on a suction filter. When completely free from acetone the powder is kept in bottles at room temperature. This material can be stored for three months without deterioration.

3. Preparation of thromboplastin for use. $-0.5 \mathrm{~g}$. of the dried powder are suspended in $5 \mathrm{ml}$. of 0.85 per cent saline and the mixture is incubated at $37^{\circ} \mathrm{C}$. for 15 minutes. The suspension is centrifuged for 5 minuies at 1,500 r.p.m. and the supernatant emulsion is diluted 1 in 10 with 0.85 per cent saline. The solution should be made up freshly as required, and this concentration was usually found to be the optimum for levels of prothrombin between 5 and 100 per cent (see below).

4. Calcium chloride solution. -2.875 g. of anhydrous calcium chloride are dissolved in $1,000 \mathrm{ml}$. of distilled water to make a 0.025 molar solution.

5. Performance of the test. $-0.1 \mathrm{ml}$. of undiluted normal plasma is added to $0.1 \mathrm{ml}$. of brain enulsion and the mixture is warmed to $37^{\circ} \mathrm{C}$. in a water bath. $0.1 \mathrm{ml}$. of $0.025 \mathrm{M}$ calcium chloride is then added rapidly from a graduated Pasteur pipette and the coagulation time is recorded from the time of addition of the calcium. The test should be made in triplicate and the mean of three readings recorded. The same procedure is repeated with the abnormal plasma. If the normal p'asma gives a clotting time of more than 30 seconds the technique is unsatisfactory.

6. The optimum concentration of brain thromboplastin and calcium

(a) Brain thromboplastin.-Aggeler and Lucia (1938) have shown that prolongation of the coagulation time occurred with both high and low concentrations of brain emulsion. With normal undiluted plasma the range within which a minimum coagulation time occurred was wide, but with pathological or diluted plasmas the optimum range was limited. Similar results were obtained in this laboratory. The optimum concentration usually corresponded to the dilution mentioned 
above, but different batches of brain vary slightly. The optimum concentration may be determined as follows: $0.5 \mathrm{~g}$. of dried brain are suspended in $5 \mathrm{ml}$. of saline and the supernatant emulsion removed as described above. This concentration is called 100 per cent, and from this a number of dilutions are made. Plasma containing various amounts of prothrombin is then prepared by dilution with saline or prothrombin-free plasma. In one experiment the curves shown in Fig. 1 were obtained.

(b) Calcium-Jaques and Dunlop (1945b) have shown that the use of an optimum concentration of calcium is important. As with brain thromboplastin the optimum concentration is much more limited with low levels of prothrombin. It was found to be $0.1 \mathrm{ml}$. of a $0.025 \mathrm{M}$ solution as used in Quick's test.

7. The expression of results.-If the clotting time of plasma is related to its prothrombin concentration the clotting time of an abnormal plasma must be compared to that of a normal before any inference can be drawn as to its relative prothrombin content. Many authors have made this comparison by dividing the clotting time of the normal plasma by that of the abnormal and expressing the result as a percentage. The " prothrombin

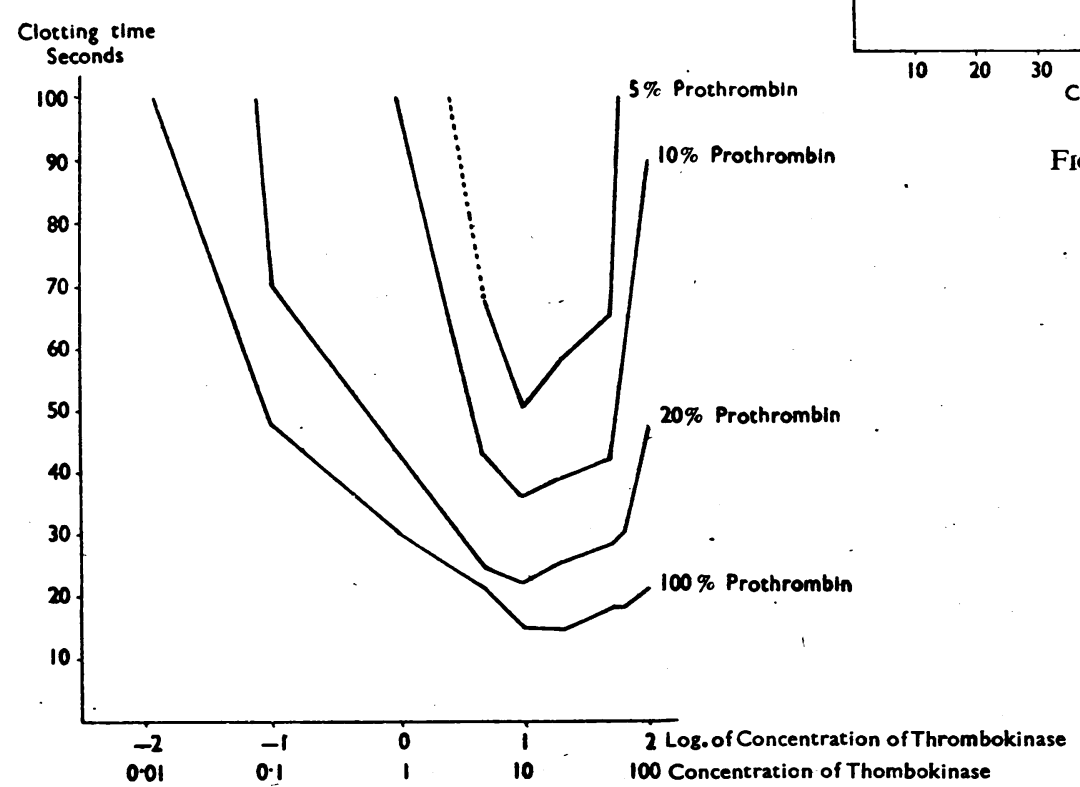

FIG. 1.-Graphs to show alteration in clotting time observed;when Quick's test is used with different concentrations of brain thromboplastin and plasma containing varying amounts of prothrombin. The prothrombin concentration was reduced by dilution with prothrombin-free plasma obtained by Seitz filtration. index "thus obtained is based on the assumption that the concentration of prothrombin bears a linear relation to the clotting time, whereas in fact the two variables are related by a hyperbolic curve. The percentage of prothrombin must therefore be read from a calibration curve made by testing

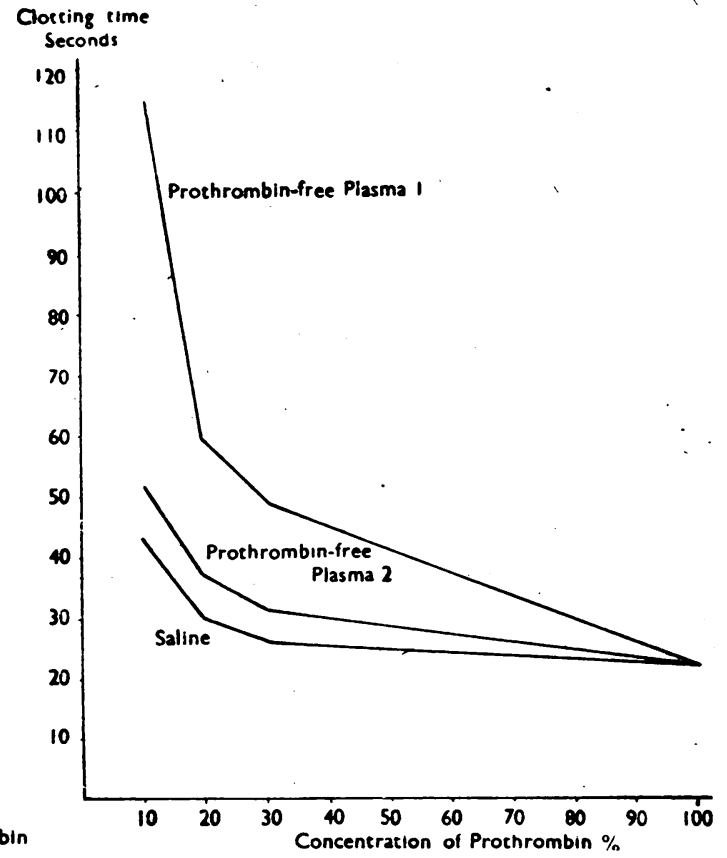

FIG. 2.-Graphs showing the alteration in the shape of the prothrombin dilution curve with different diluents using brain thromboplastin. Prothrombin-free plasma 1 was prepared from blood collected in a silicone coated container. The plasma was obtained after centrifuging for 30 minutes at 20,000 r.p.m., and the prothrombin was removed by adsorption with an excess of freshly precipitated barium sulphate. Prothrombin-free plasma 2 was prepared from blood collected in an ordinary glass container. The plasma was obtained after centrifuging for 5 minutes at 1,500 r.p.m., and the prothrombin was removed by adsorption with the minimum amount 0 freshly precipitated barium sulphate. 
samples of normal plasma in which the prothrombin concentration has been reduced artificially. The determination of prothrombin by this technique would be relatively simple if a single reference curve could be drawn and the same curve were used by all workers. Unfortunately this is not possible because it is customary to reduce the prothrombin content by simple dilution. This procedure certainly effects a quantitative reduction in prothrombin but unfortunately also reduces substances other than prothrombin which may be active in prothrombin conversion. Thus, if saline is used platelets and fibrinogen are lowered, and if prothrombin-free plasma is used platelets and possibly accessory factors concerned in prothrombin conversion are reduced. As Conley and Morse (1948) have shown, the reference curves vary in shape according to the diluent used, and one clotting time may be taken to represent a number of different concentrations of prothrombin according to which curve is selected as a standard (Fig. 2).

A further complication is introduced by the fact that different batches of brain thromboplastin give different coagulation times with the same normal undiluted plasma; thus using any one diluent it is necessary to prepare a number of curves at different levels of thromboplastin activity.

Since all of the diluents produce artificial mixtures not comparable to naturally occurring prothrombin-deficient plasmas, a selection between them is arbitrary. In practice we have chosen to use saline because it gives readily reproducible results in normal plasma and because of the simplicity of the processes involved. For standard reference curves a series of saline dilution curves were made from normal plasma using different batches of brain thromboplastin which gave coagulation times from 14 to 25 seconds with undiluted plasma (Fig. 3).

It was found convenient to use a simple mathematical conversion to straight lines in place of the curves because with these it is easy to interpolate for intermediate values. This conversion is achieved by plotting the clotting time against the reciprocal

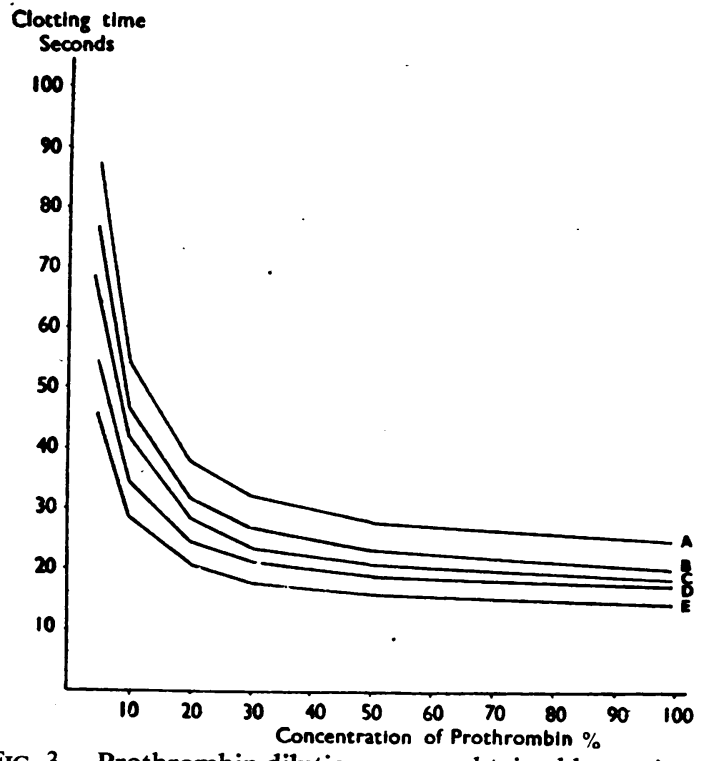

FIG. 3.-Prothrombin dilution curves obtained by testing the prothrombin time of saline dilutions of normal plasma using different batches of brain thromboplastin of varying potency.

of the concentration of prothrombin (Fig. 4).

Example.-Coagulation time of normal plasma: 18 seconds. Coagulation time of abnormal plasma: 42 seconds. Curve $\mathrm{C}$ in Fig. 4 is selected because the undiluted plasma (100 per cent) gives coagulation time of 18 seconds. Drawing a line parallel to the

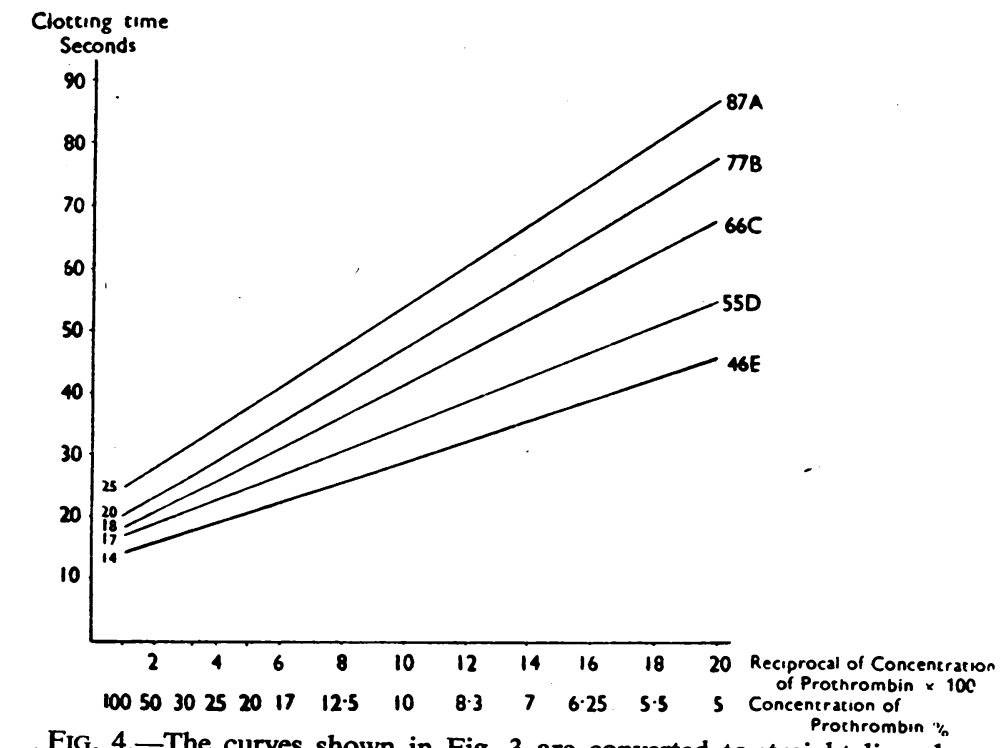

FIG. 4.-The curves shown in Fig. 3 are converted to straight lines by plotting the clotting time against the reciprocal of the concentration 
ordinate through 42 seconds it will be seen that this Concentration of crosses the selected curve at a level of prothrombin Prothrombin \% equivalent to a dilution of 10 per cent in the normal plasma ; the plasma would therefore be said to contain 10 per cent of prothrombin.

This method of standardization is clearly quite arbitrary, and while the results with any one set of calibration curves will be comparable, the results from different hospitals can be related only if the reference curves are given. In much of the published work this has not been done.

\section{(b) WrTts and Hobson's Technique}

The technique is essentially similar to that of Quick except that brain thromboplastin is replaced by a 1 in 10,000 solution of Russell's viper venom (Burroughs Wellcome's "Stypven") to $1 \mathrm{ml}$., of which $0.1 \mathrm{ml}$. of a 1 per cent alcoholic solution of crude lecithin is added. The saline dilution curves in normal plasmas - are remarkably constant, and in most cases one curve will suffice for the expression of results as percentage of prothrombin. However, as with brain thromboplastin, different diluents give different curves (Fig. 5).

\section{Case Reports}

A. Cases in which the Percentage of Prothrombin was Estimated by WITtS aND Hobson's TECHNIQUE

Case 1.-Mr. W.W., aged 53, was treated with dicoumarin for thrombophlebitis of the leg. $1,100 \mathrm{mg}$. of dicoumarin were given in the first eight days and no significant fall in prothrombin was recorded until

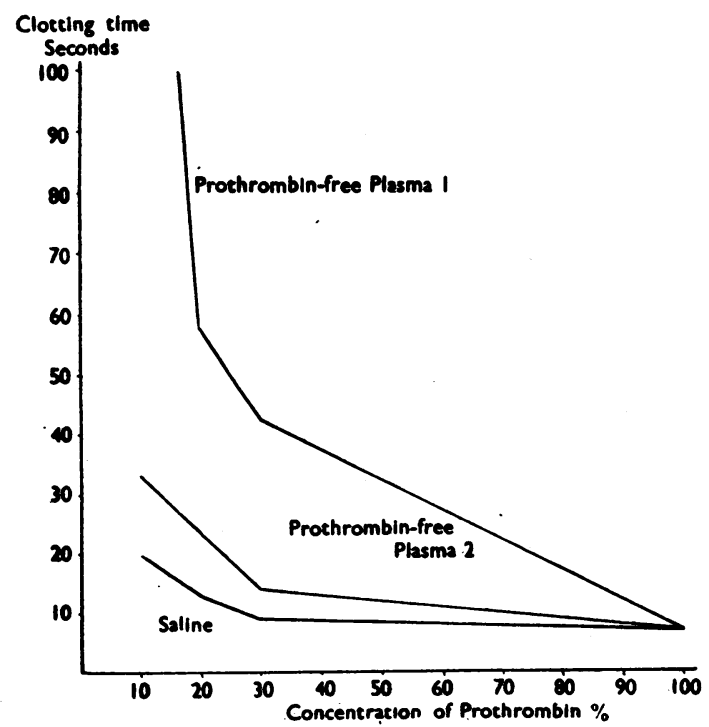

FIG. 5.-Graphs to show the alteration in the shape of the prothrombin dilution curves with different diluents using Russell's viper venom and lecithin as thromboplastin. Prothrombin-free plasmas 1 and 2 are the same as those of Fig. 2.

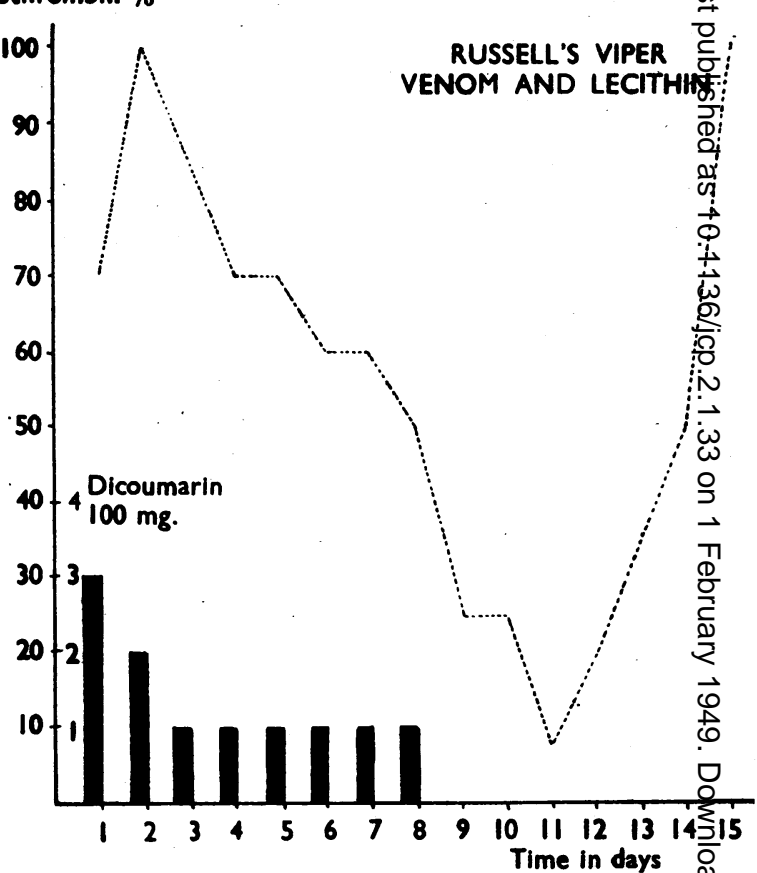

FIG. 6.-Diagram to show the alteration in concentration of prothrombin, as estimated with Russell's viper. venom, in relation to the dosage of dicoumarin in $\vec{\overrightarrow{ }}$ Case 1

the ninth day, when there was a fall to 25 per cent and a low level was maintained for four days (Fig. 6).?

Case 2.-Mrs. C.D., aged 44, was treated with dicoumarin for thrombophlebitis of the leg with:pulmonary embolus following a hysterectomy for 3 fibroid. $1,100 \mathrm{mg}$. of dicoumarin were given in the first eleven days and a significant fall of prothrombin was delayed until the tenth day, when readings of $14, ₹$ 30 , and 15 per cent were recorded on three consecutive days (Fig. 7).

Case 3.-Mrs. E.C., aged 53, was given dicoumarin following operation for prolapse to prevent the occurrence of femoral thrombosis. She was given $700 \mathrm{mg}$. $O 5$ dicoumarin in the first four days, and on the fifth day N had severe haemorrhage from the operation site which necessitated transfusion of 2 pints of blood. The $\omega$ surgeons could find no local cause for this bleeding 0 Her prothrombin time remained within normal limitso throughout.

These cases followed a course similar to that described by Wright and Prandoni (1942), who used Russell's viper venom for prothrombin esti-苂

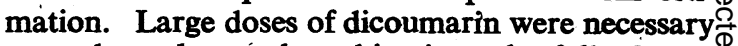
to prolong the prothrombin time, the fall of pro- $\varrho$ thrombin was delayed, and unpredictable haemor rhage occurred. 


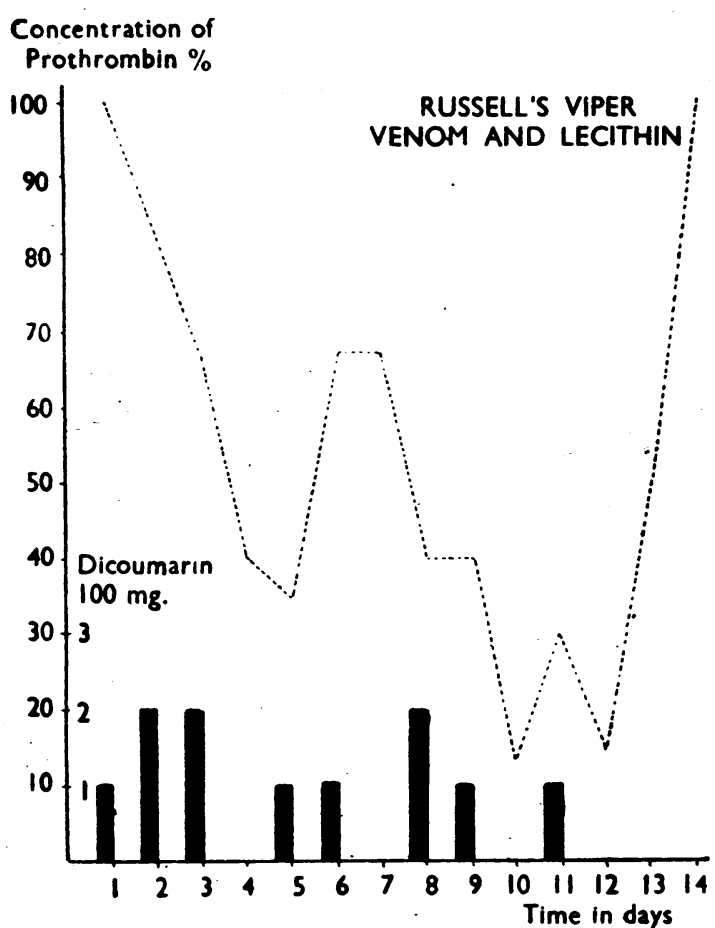

Fig. 7.-Diagram to show the alteration in concentration of prothrombin, as estimated with Russell's viper venom, in relation to the dosage of dicoumarin in Case 2.

\section{B. Cases in which the Prothrombin was Estimated BY BOTH WITTS AND HOBSON's AND QUICK's TECHNIQUes}

Case 4.-Mr. E. W., aged 59, suffered from cardiac asthma and was treated with dicoumarin for thrombophlebitis of the leg. This patient was admitted to hospital twice for recurrence of this condition. On the first admission his prothrombin time was estimated by Witts and Hobson's technique. In nineteen days he was given $2,000 \mathrm{mg}$. of dicoumarin, and on one occasion only was there any appreciable lengthening of the prothrombin time. On the second admission three months later he was given $600 \mathrm{mg}$. of dicoumarin in the first three days and showed lengthening of prothrombin time by Quick's technique on the third day. The clotting time was considerably prolonged for four days and gradually returned to normal in the course of the next four days.

Case 5.-Mrs. H.F., aged 39, was treated with dicoumarin for puerperal thrombophlebitis of the leg. In this patient $900 \mathrm{mg}$. dicoumarin were given in twelve days, and on some days Witts and Hobson's technique was used and on others Quick's technique. There was a marked lack of correspondence between the results of the two methods (Fig. 8).
These cases suggest that Witts and Hobson's technique is far less sensitive than that of Quick in recording the hypoprothrombinaemia of dicoumarin therapy. In order to substantiate this probability the course of the prothrombin was followed by both techniques simultaneously in the next three cases.

Case 6.-Mr. H., aged 71, was treated with dicoumarin for thrombophlebitis following a cystotomy for benign prostatic hypertrophy. He was given $500 \mathrm{mg}$. of dicoumarin in the first two days. By Quick's technique the prothrombin dropped suddenly to 9 per cent on the third day and fell to 5 per cent on the fifth day, when there was a slight urinary haemorrhage. He was given vitamin $K$, the haemorrhage ceased rapidly, and the prothrombin returned to normal in four days. By Witts and Hobson's technique the prothrombin was significantly abnormal on two days only, and at the time of haemorrhage a level of 35 per cent was recorded (Fig. 9).

Case 7.-Mrs. F., aged 73, was treated with dicoumarin for thrombophlebitis of the leg following operation of the left obturator nerve. She was given $800 \mathrm{mg}$. during the first thirteen days, and the relative sensitivity of Witts and Hobson's and Quick's techniques is shown in Fig. 10.

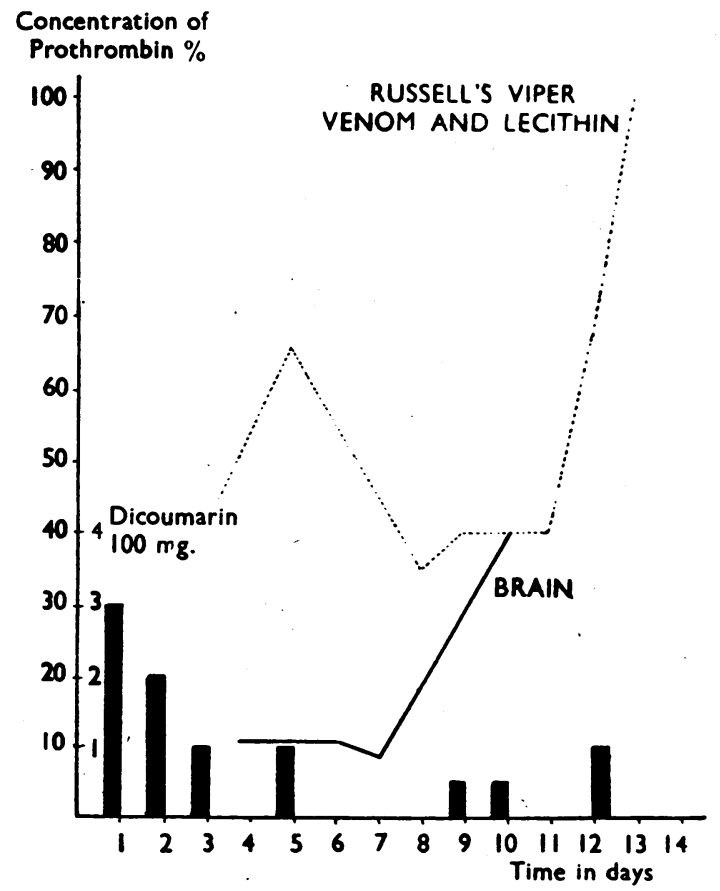

FIG. 8.-Diagram to show the alteration in concentration of prothrombin, as estimated with brain thromboplastin and Russell's viper venom and lecithin, in relation to the dosage of dicoumarin in Case 5. 
Case 8.-Mr. D., aged 45, was given dicoumarin Prothrombin Concentration

for treatment of pulmonary thrombosis following appendicectomy. $500 \mathrm{mg}$. were given in the first three days, and the patient showed an unusually marked response to the drug when the prothrombin was estimated with a brain thromboplastin (Fig. 11). With Russell's viper venom and lecithin the response was delayed and was less in extent.

The Cause of the Discrepancy between the Prothrombin Percentages Recorded with Russell's Viper Venom and Lecithin and Brain Thromboplastin

Using a two-stage technique the percentage of prothrombin estimated by the two types of thromboplastin is approximately the same (Table I). The discrepancy cannot therefore be accounted for by differences in the amount of thrombin formed. The clotting times measured by the onestage technique are converted to so-called "percentage of prothrombin" by means of artificial dilution curves. Since the clotting times recorded by the same pathological plasma give different
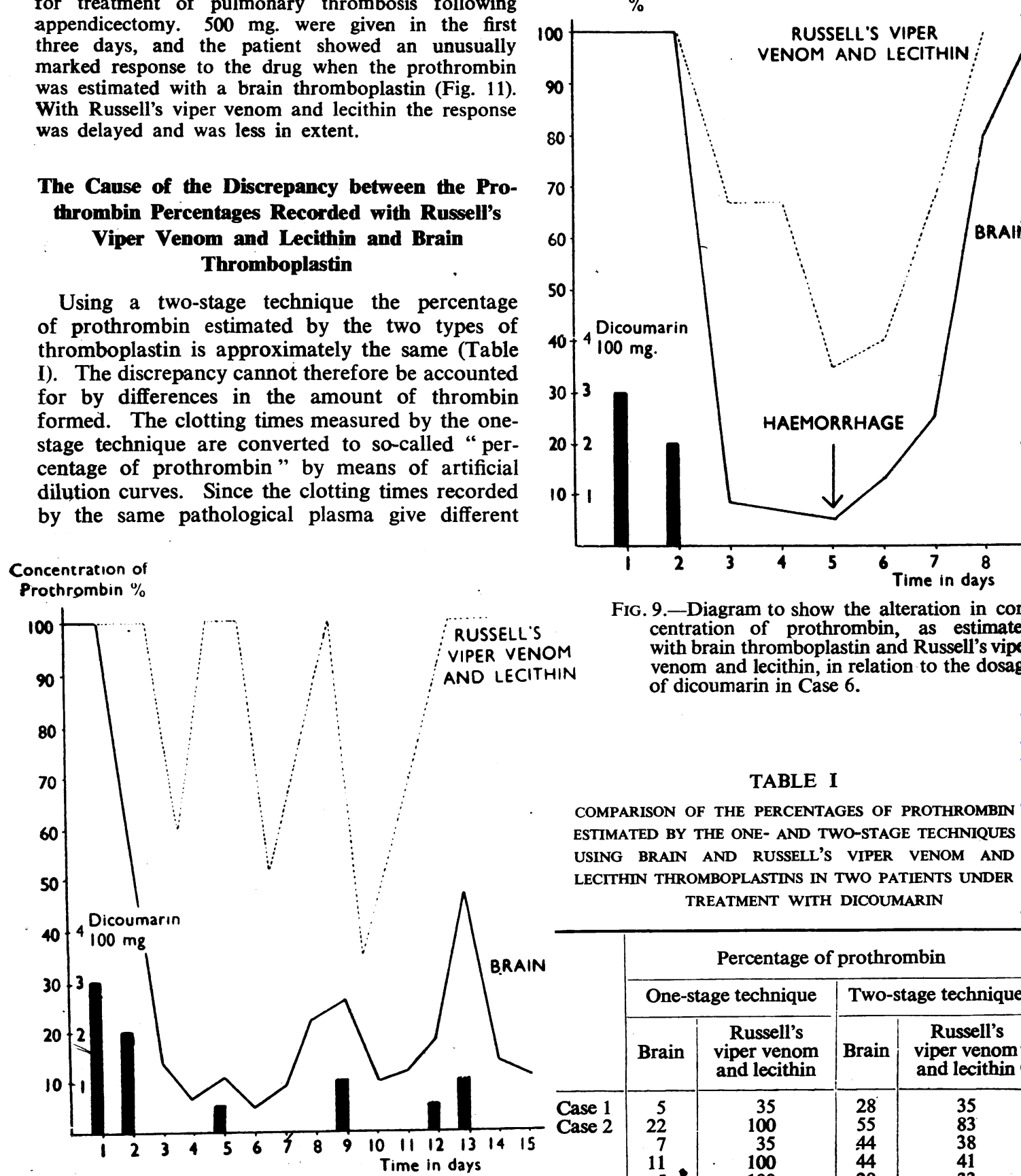

FIG. 9.-Diagram to show the alteration in cono centration of prothrombin, as estimatec with brain thromboplastin and Russell's viperi venom and lecithin, in relation to the dosage of dicoumarin in Case 6.

TABLE I

COMPARISON OF THE PERCENTAGES OF PROTHROMBIN ESTIMATED BY THE ONE- AND TWO-STAGE TECHNIQUES USING BRAIN AND RUSSELL'S VIPER VENOM AND LECITHIN THROMBOPLASTINS IN TWO PATIENTS UNDER TREATMENT WITH DICOUMARIN

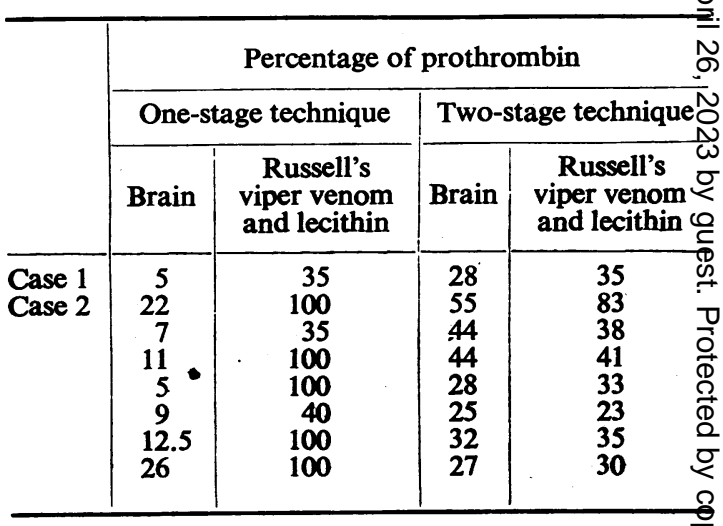
prothrombin, as estimated with brain thromboplastin and Russell's viper venom and lecithin, in relation to the dosage of dicoumarin in Case 7. 
percentages of prothrombin with the two types of thromboplastin, it is difficult to avoid the conclusion that the dilution curves are at fault. It has already been noted that all the diluents used reduce factors other than prothrombin, and it seems possible that one or more of these factors may have a greater effect on the speed of prothrombin conversion with Russell's viper venom and lecithin than with brain thromboplastin.

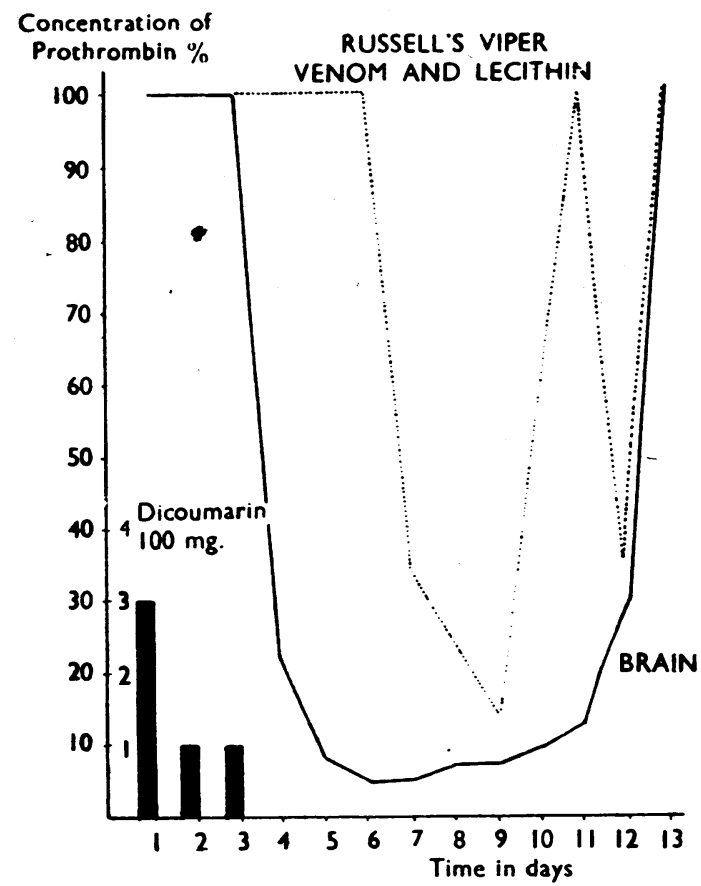

FIG. 11.-Diagram to show the alteration in concentration of prothrombin, as estimated with brain thromboplastin and Russell's viper venom and lecithin, in relation to the dosage of dicoumarin in Case 8 .

This hypothesis can be tested if normal plasma is diluted with naturally occurring prothrombindeficient plasma in which it is assumed that only prothrombin is reduced. Curves constructed from the coagulation times of various mixtures of normal plasma with prothrombin-deficient plasmas are shown in Figs. 12 and 13 . Fig. 12 was derived from a vitamin-K deficient plasma which showed only a trace of prothrombin, and in Fig. 13 a dicoumarin plasma was used which was found to contain 18 per cent of prothrombin by Quick's technique.

It will be seen that when Russell's viper venom and lecithin is used mixtures of normal plasma with naturally occurring prothrombin-deficient

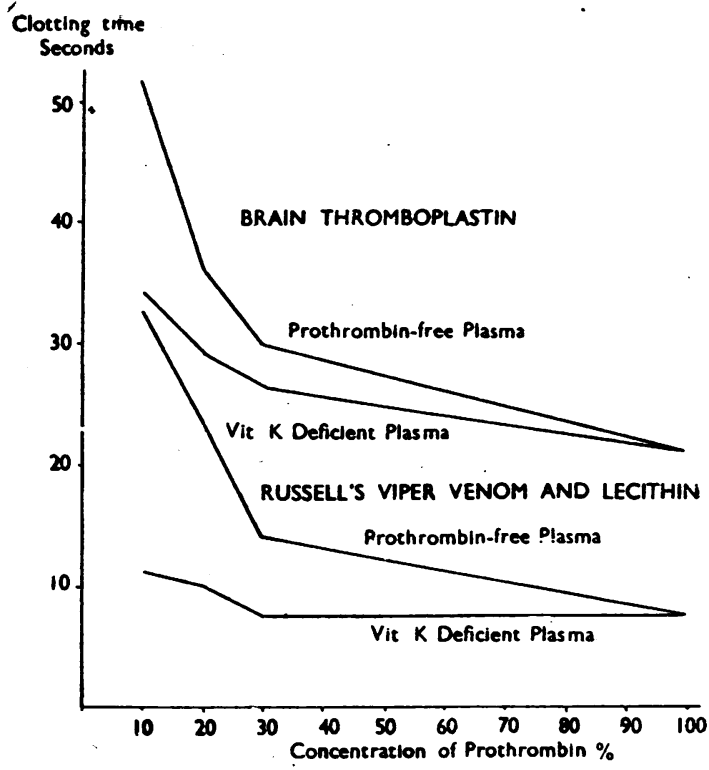

Fig. 12.-Curves to show the difference between the prothrombin dilution curves obtained by the dilution of normal with $(a)$ vitamin-K deficient plasma, $(b)$ prothrombin-free plasma, using brain and Russell's viper venom and lecithin as thromboplastins. The prothrombin-free plasma was made by adsorption with barium sulphate.

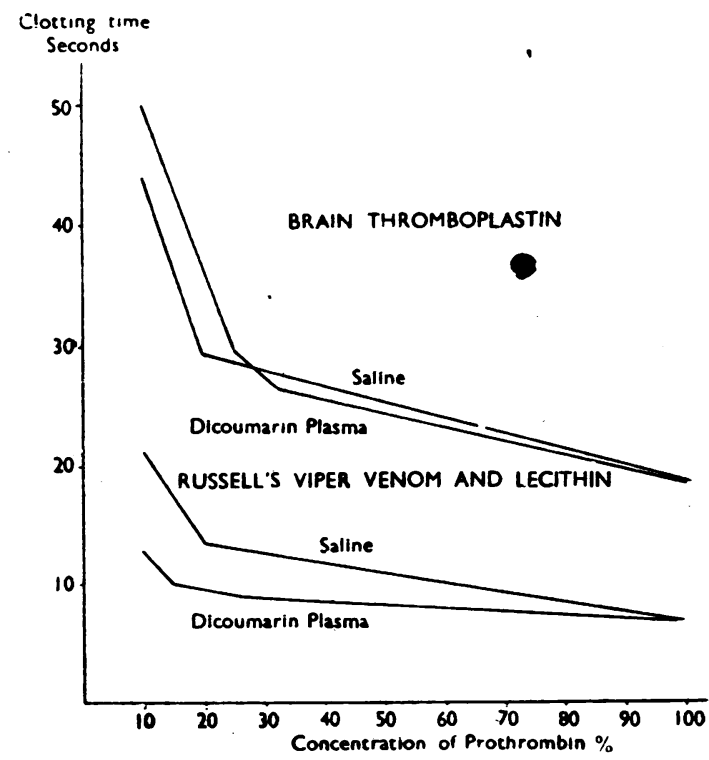

FIG. 13.-Curves to show the difference between the prothrombin dilution curves obtained by dilution of normal plasma with $(a)$ dicoumarin plasma, and $(b)$ saline, using brain and Russell's viper venom as thromboplastins. 


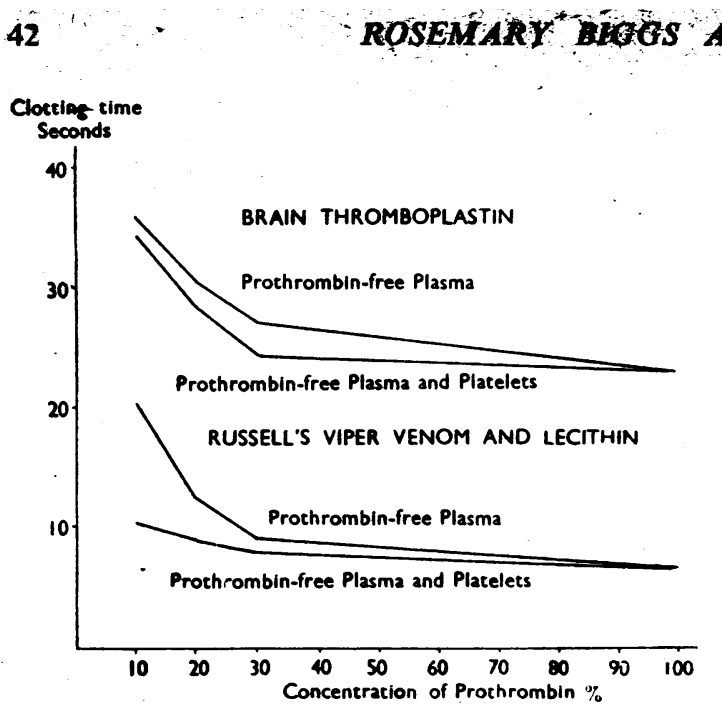

Fig. 14.-Curves to show the difference between the prothrombin dilution of normal plasma with $(a)$ prothrombin-free plasma, (b) prothrombin-free plasma with the addition of platelets to correspond with original concentration in the plasma, using brain and Russell's viper venom and lecithin as thromboplastins. The prothrombin-free plasma was obtained by adsorption with barium sulphate.
In fact this thromboplastin appears to measure a reduction in platelets as much as the fall in prothrombin concentration.

The extraordinary lack of sensitivity of Russell's viper venom and lecithin thromboplastin to changes in prothrombin concentration probably lies in the mechanism of thrombin generation. From the reaction of normal plasma to thrombin it can be calculated that only 5 per cent of the available thrombin that could be formed by a normal plasma is necessary for its coagulation, and it is the speed with which this level of thrombin is reached that controls the one-stage coagulation time. In Fig. 15 the early stages of thrombin generation have been followed in a normal plasma and in the plasma of a patient under treatment with dicoumarin. The minimum letel of thrombin for rapid coagulation of the plasma has been indicated. With brain thromboplastin thrombin formation follows an autocatalytic type of curve, and alteration in prothrombin concentration greatly increases the time necessary for the generation of a minimum amount of thrombin. With Russell's viper venom and lecithin, on the other plasma give much shorter coagulation times than are recorded by corresponding mixtures with saline or artificial prothrombin-free plasma. Moreover, the alteration in clotting time between 100 and 10 per cent becomes immeasurably small, giving a total range of clotting times from 7 to 11 seconds. With brain thromboplastin the discrepancy is much less marked.

The next problem that arises is which of the plasma constituents other than prothrombin is responsible for the discrepancy. One factor which is easily tested is the presence of platelets. It will be seen from Fig. 14 that when Russell's viper venom and lecithin thromboplastin is used the addition of platelets to mixtures of normal and prothrombin-free plasma causes a reduction in the coagulation time which is particularly marked at low levels of prothrombin.

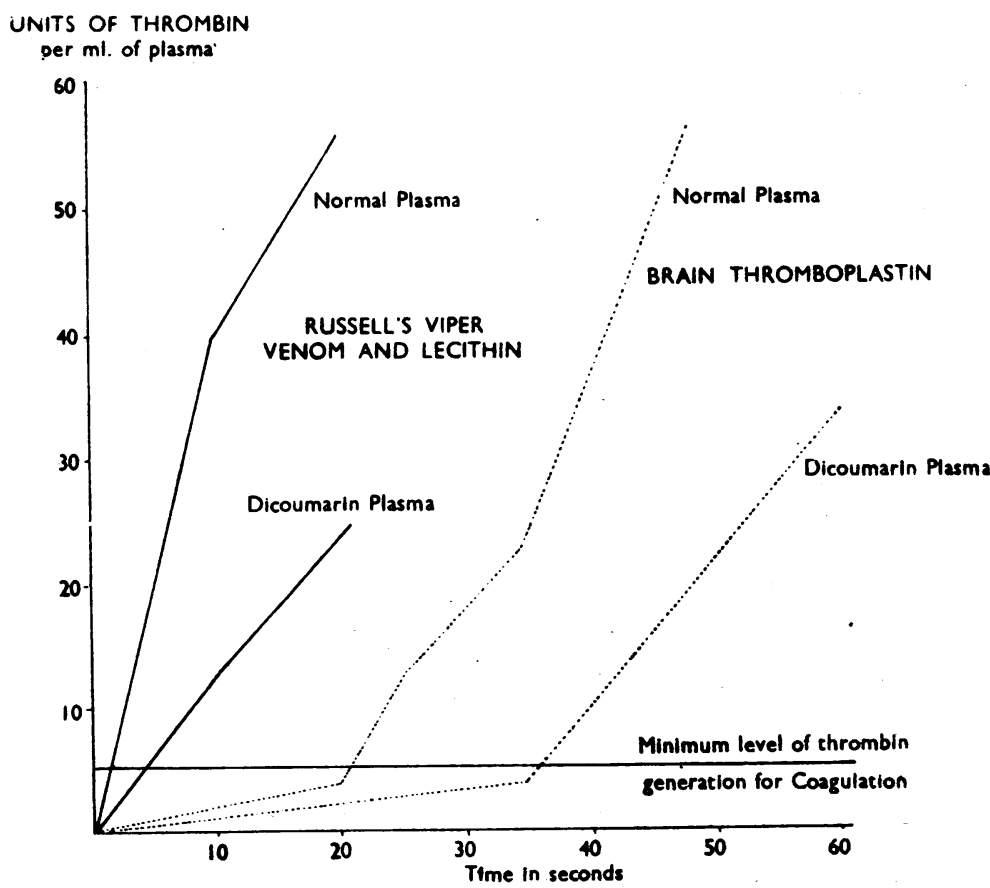

FIG. 15. - Curves to show the early stage of thrombin generation in normal and dicoumarin plasma using a two-stage technique with (a) brain thromboplastin, and (b) Russell's viper venom and lecithin. The probable minimum level of thrombin for coagulation of the plasma in less than 10 seconds is indicated. The thrombin units are calculated to correspond with $1 \mathrm{ml}$. of the original plasma. 
hand, an immediate liberation of thrombin occurs and the minimum level of thrombin is attained rapidly even at low prothrombin concentrations. Reduction in prothrombin content therefore makes little difference to the one-stage prothrombin time. Moreover, with Russell's viper venom and lecithin the generation of thrombin is so rapid that the sensitivity of the one-stage technique is almost certainly limited by the speed of the thrombin fibrinogen reaction.

If the discrepancy between Russell's viper venom and lecithin and brain thromboplastin results from a differential sensitivity to factors other than prothrombin, a similar difference should occur in all types of hypoprothrombinaemia. In a limited series of pathological cases such a discrepancy was found (Table II).

TABLE II

PERCENTAGE OF PROTHROMBIN ESTIMATED WITH BRAIN THROMBOPLASTIN AND RUSSELL'S VIPER VENOM AND LECITHIN IN VARIOUS PATHOLOGICAL CONDITIONS

\begin{tabular}{|c|c|c|}
\hline \multirow[b]{2}{*}{ Condition } & \multicolumn{2}{|c|}{ Prothrombin concentration } \\
\hline & $\begin{array}{c}\text { Using brain } \\
\text { thrombo- } \\
\text { plastin } \\
(\%)\end{array}$ & $\begin{array}{l}\text { Using Russell's } \\
\text { viper venom } \\
\text { and lecithin } \\
\text { \% }\end{array}$ \\
\hline $\begin{array}{l}\text { Cirrhosis of the liver } \\
\text { ", } \\
\text { Vitamin ", } \\
\text { ", deficiency } \\
\text { Ulcerative colitis } \\
\text { Treatment with quini- } \\
\text { dine sulphate }\end{array}$ & $\begin{array}{c}35 \\
14 \\
35 \\
50 \\
11 \\
20 \\
25 \\
\text { Clotting time } \\
20 \text { min. } \\
25 \\
30\end{array}$ & $\begin{array}{c}30 \\
50 \\
80 \\
100 \\
35 \\
80 \\
100 \\
\text { Clotting time } \\
56 \text { sec. } \\
35\end{array}$ \\
\hline
\end{tabular}

\section{Discussion}

Much of the confusion aroused by the estimation of prothrombin follows from an unwarranted belief that the one-stage test can give a real measure of prothrombin concentration provided that sufficient care is taken in standardization of the technique. From the experiments described here it is clear that this is not possible. Not only do the two thromboplastins, each carefully standardized, give different results, but each thromboplastin can be made to give a series of differing results according to which of the diluents is used to prepare the calibration curves. The technique cannot therefore measure prothrombin, but gives a relative measure of the speed of production of the small amount of thrombin necessary for coagulation. It might save confusion if all pretence at measuring "prothrombin percentage" were abandoned and the results were expressed as "relative prothrombin efficiency" or, as was suggested by Witts (1942) and Witts and Hobson (1942), an "accelerated clotting time" in percentage of normal.

The value of the test depends essentially on its ability to predict a haemorrhagic level. Bollman and Preston (1942) have shown that haemorrhage following dicoumarin administration is not directly proportional to the "concentration of prothrombin." In the patients described here it is clear that Cases 3 and 6 had a particular predisposition to the haemorrhage. Fortunately haemorrhage often fails to occur with a very low content of prothrombin, and Barker and others (1945) have claimed that haemorrhage was uncommon with prothrombin levels above 10 per cent by Quick's technique. In Case 6 the "prothrombin concentration" was 5 per cent at the time of bleeding, and haemorrhage rapidly ceased when it was raised above 10 per cent. With Quick's technique, therefore, it is probable that if the "concentration of prothrombin " is maintained above 10 per cent no uncontrollable haemorrhage will occur. The value of this test rests on the vast clinical material in which it has been used rather than on the soundness of its theoretical foundations.

With Russell's viper venom and lecithin, on the other hand, no certain haemorrhagic level can be defined because a sufficient number of cases have not been recorded for any such conclusion to be reached. Moreover, the technique is dangerously insensitive to alterations in prothrombin concentration, and in a routine laboratory it is impossible to measure the one or two seconds differences in coagulation time that may bring the patient within the haemorrhagic level.

It is surprising that in an annotation in the British Medical Journal (1947) doses of 100 to 300 mg. of dicoumarin daily are recommended, and in a further annotation (1948) mention is made of the daily administration of $200 \mathrm{mg}$. These amounts are dangerously high and would certainly cause haemorrhage in patients such as Cases 3 and 6 with a predisposition to bleeding.

\section{Summary}

1. Russell's viper venom with lecithin is frequently used to replace brain thromboplastin for the estimation of prothrombin in the one-stage technique. 
2. The assumption that Russell's viper venom and lecithin will give the same results as brain thromboplastin is not justified by theoretical considerations or by experience of its use in practice.

3. One pathological plasma will record a different percentage of prothrombin when tested with brain and Russell's viper venom thromboplastins even when the results are read from appropriate calibration curves.

4. The calibration curves are drawn from artificial plasma mixtures which are not comparable to naturally occurring prothrombin-deficient plasmas. The two thromboplastins have a different sensitivity to the reduction of factors other than prothrombin which are lowered in the artificial mixtures.

5. The one-stage technique using brain thrombopiastin is of proved value but cannot measure prothrombin concentration, and its results would be better recorded as "relative prothrombin efficiency" or as an "accelerated clotting time" expressed as a percentage of normal.

6. Russell's viper venom and lecithin cannot be used to replace brain thromboplastin as a routine procedure because it is not sufficiently sensitive to alterations in prothrombin concentration.

We should like to thank Mr. J. Pilling, B.Sc., who carried out much of the laboratory work.

\section{REFERENCES}

Aggeler, P. M., and Lucia, S. P. (1938). Proc. Soc. exp. Biol. N.Y., 38,11 .

Allen, E. V. (1947). J. Amer. med. Ass., 134, 323.

Annotation (1947). Brit. med. J., 2, 662.

Annotation (1948). Brit. med. J., 1, 988.

Barker, N. W., Allen, E. V., and Waugh, J. M. (1943). Proc. Mayo Clin., 18, 102 .

Barker, N. W., Cromer, H. E., Hurn, M., and Waugh, J. M. (1945). Surgery, 17, 207.

Bingham, J. B., Meyer, O. O., and Howard, B. (1943). Amer. J. med. Sci., 205, 587 . Bingham, J. B., Meyer, O. O., and Pohle, F. J. (1941). Amer. J. med.

Bollman, J. L., and Preston, F. W. (1942). J. Amer. med. Ass., 120 1021.

Butsch, W. L., and Stewart, J. D. (1942). Arch. Surg., 45, 551.

Canti, G., and Robertson, D. J. (1948). Brit. med. J., 1, 125.

Cleland, G. (1947). Brit. med. J., 2, 748.

Conley, C. L., and Morse, W. I. (1948). Amer. J. med Sci., 215, 158 으

Cotlove, E., and Vorzimer, J. J. (1946). Ann. intern. Med., 24, 648.

de Beer, E. J. (1947). J. Lab. clin. Med., 32, 90.

Fullerton, H. W. (1940). Lancet, 2, 195 .

Gefter, W. I., Kramer, D. W., and Reinhold, J. G. (1944). Amer

Heart J., 28, 321.
Glueck, H. I., Strauss, V., Pearson, J. S., and McGuire, J. (1948). Amer. Heart J., 35, 269.

Hobson, F. C. G., and Witts, L. J. (1941). J. Path. Bact., 52, 367. .

James, G. A. (1948). Brit. med. J., 1, 475.

Jaques, L. B., and Dunlop, A. P. (1945a). Amer. J. Physiol., 143, $355 \vec{\omega}$

Jaques, L. B., and Dunlop, A. P. (1945b). Amer. J. Physiol., 145, 67.

Lamb, G. (1903). Sci. Mem. Med. San. Depts., India. New Series No. 4.

Leathes, J. B., and Mellanby, J. (1939). J. Physiol., 96, 38 P.

Lempert, H. (1948). Brit. med. J., 1, 125. and its Failure in the Haemorrhagic States." Thesis for thecu degree of Doctor of Medicine, University of London.

Macfarlane, R. G and Barnet, B. (1934). Lancet, 2, 985.

Macfarlane, R. G., Trevan, J. W., and Attwood, A. M. P. (1941). 의 J. Physiol., 99, 7.

Marsh, F. (1947). Brit. med. J., 2, 1009

Marsh, F. (1948). Brit. med. J., 1, 319.

Martin, C. J. (1894). J. Physiol., 15, 380.

Mellanby, J. (1909). J. Physiol., 38, 441.

Meyer, O. O., Bingham, J. B., and Axelrod, V. H. (1942). Amer. J. med. Sci., 204, 11.

Morawitz, P. (1905). Ergebn. Physiol., 4, 307.

Page, R. C., and de Beer, E. J. (1942-3). J. Lab. clin. Med., 28, $912 . \rightarrow$

Page, R. C., and de Beer, E. J. (1943). Amer. J. med. Sci., 206, 336.

Page, R. C., de Beer, E. J., and Orr, M. L. (1941-2a). J. Lab. clin. Med., 27, 197.

Page, R. C., de Beer, E. J., and Orr, M. L. (1941-2b). J. Lab. clin. Med., $27,830$.

Page, R. C., and Russell, H. K. (1941). J. Lab. clin. Med., 26, $1366 . \mathrm{O}^{\mathrm{O}}$

Pivawer, M. J. (1947). Brit. med. J., 2, 928.

Quick, A. J. (1942). "The Hemorrhagic Diseases and the Physiologyo of Hemostasis." Charles C. Thomas. Illinois.

Quick, A. J. (1945). J. biol. Chem., 161, 33.

Quick, A. J. (1947). Amer. J. Physiol., 151, 63.

Shapiro, S. Redish, M. H. and Campbell, H. A. (1943). Proc. Soc exp. Biol. N.Y., 52, 12 .

Shapiro, S., and Sherwin, B. (1943). N.Y. State J. Med., 43, 45

Shapiro, S., Sherwin, B., Redish, M., and Campbell, H. A. (1942). Proc. Soc. exp. Biol. N.Y., 50, 85.

Shapiro, S., Sherwin, B., and Gordimer, H. (1942). Ann. Surg. 116, 175

Schmidt, A. (1895). “Weitere Beitrage zur Blutlehre." Bergmann

Wiesbaden.
Trevan, J. W., and Macfarlane, R. G. (1936). Med. Res. Coun.? Ann. Rep., p. 143.

Witts, L. J. (1942). Glasg. med. J., 137, 57.

Witts, L. J., and Hobson, F. C. G. (1940). Brit. med. J., 2, 862.

Witts, L. J., and Hobson, F. C. G. (1942). Brit. med. J., 1, 575.

Wright, I. S., and Prandoni, A. (1942). J. Amer. med. Ass., 120, 1015 . 